\title{
MlaC represents a unique class of solute-binding proteins
}

\author{
Angshu Dutta, Shankar Prasad Kanaujia \\ Dept. of Biosciences and Bioengineering, Indian Institute of Technology Guwahati, Guwahati, India \\ spkanaujia@gmail.com
}

In Gram-negative bacteria, the maintenance of lipid asymmetry (Mla) system is involved in the transport of phospholipids (PLs) between the inner membrane (IM) and outer membrane (OM), thereby maintaining OM asymmetry [1]. The Mla system is a multicomponent intermembrane machinery which is composed of three main constituents- an OM MlaA-OmpC/F complex, a free-floating periplasmic protein $\mathrm{MlaC}$ and an IM ATP-binding cassette (ABC) transporter complex MlaFEDB [2]. MlaC, which serves as the solute-binding protein (SBP), has been reported to have atypical structural features [3]. However, an in-depth investigation highlighting the peculiarities and the mechanism of ligand binding is still lacking. This study reports, for the first time, the crystal structure of MlaC from Escherichia coli at a resolution of 2.5 $\AA$ in a quasi-open state and in complex with a PL. The analysis reveals that MlaC comprises two major domains viz, NTF2-like (D1) and AAA helical-bundle (D2). Each domain can be divided into two subdomains (D1R1, D1R2; D2R1, D2R2) that are arranged in a discontinuous fashion. Further, MlaC would follow a reverse mechanism of binding pocket opening and the subdomains exhibit specific movements that aid in ligand binding and orientation. Based on extensive structural analysis, a novel mechanism of ligand binding is proposed that has not been observed for any known SBP till date. Additionally, the study also highlights the unique ancestries of MlaC and other atypical SBPs that are involved in OM biogenesis. The work firmly establishes MlaC to be a one-of-a-kind transporter protein that plays critical role in maintaining OM asymmetry.

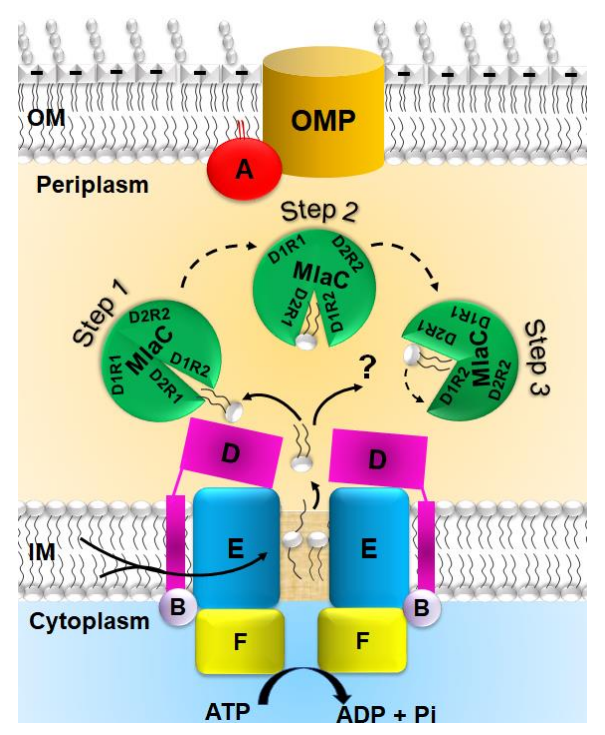

Figure 1. Proposed model of PL transport.

[1] Malinverni, J. C. \& Silhavy, T. J. (2009). Proc. Natl. Acad. Sci. U.S.A. 106, 8009.

[2] Coudray, N., Isom, G. L., MacRae, M. R., Saiduddin, M. N., Bhabha, G. \& Ekiert, D.C. (2020). Elife 9, e62518.

[3] Yero, D., Díaz-Lobo, M., Costenaro, L., Conchillo-Solé, O., Mayo, A., Ferrer-Navarro, M., Vilaseca, M., Gibert, I. \& Daura, X. (2021). Commun. Biol. 4, 1.

Keywords: ABC transporter; membrane asymmetry; phospholipid; transport

Acknowledgements: This work is supported by Science and Engineering Research Board (SERB), Department of Science and Technology, Government of India (Grant/Award Number: ECR/2018/000013). AD acknowledges the Ministry of Human Resource and Development (MHRD), Government of India. 